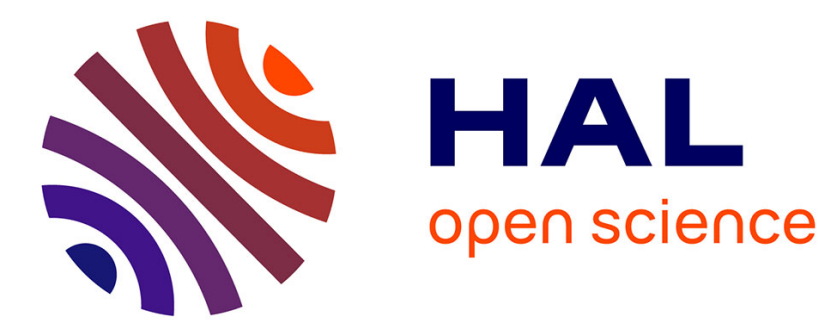

\title{
La dénotation spatiale des noms d'événements
}

Richard Huyghe

\section{To cite this version:}

Richard Huyghe. La dénotation spatiale des noms d'événements. Lingvisticae Investigationes, 2011, 34, pp.138-155. hal-00975569

\section{HAL Id: hal-00975569 \\ https://hal-univ-paris.archives-ouvertes.fr/hal-00975569}

Submitted on 8 Apr 2014

HAL is a multi-disciplinary open access archive for the deposit and dissemination of scientific research documents, whether they are published or not. The documents may come from teaching and research institutions in France or abroad, or from public or private research centers.
L'archive ouverte pluridisciplinaire HAL, est destinée au dépôt et à la diffusion de documents scientifiques de niveau recherche, publiés ou non, émanant des établissements d'enseignement et de recherche français ou étrangers, des laboratoires publics ou privés. 
Huyghe, Richard. 2011. La dénotation spatiale des noms d'événements. Lingvisticae Investigationes 34, 138-155.

\section{Résumé - La dénotation spatiale des noms d'événements}

Ce travail porte sur les propriétés descriptives spatiales des noms d'événements [Nev], souvent ignorées dans les travaux linguistiques sur l'espace. Les Nev dénotent des entités spatiales, en ce sens qu'ils peuvent s'employer comme cibles dans des phrases de localisation spatiale (Il y a une cérémonie dans l'église). Les $\mathrm{Nev}$ se distinguent toutefois des noms d'entités spatiales prototypiques par plusieurs aspects. Ils ne se combinent pas avec les compléments d'étendue spatiale (*une cérémonie de deux hectares) et se construisent avec des noms et des verbes de localisation distinctifs (le lieu / *la place de la cérémonie, Une cérémonie a lieu / *se trouve dans l'église). L'hypothèse défendue est que l'originalité de la dénotation spatiale des Nev est due à leur relation directe avec le temps. La dépendance entre les propriétés spatiales et temporelles des Nev apparaît dans leur emploi en position de site (Pierre se rend à la cérémonie). D'une part, les sites spatiaux événementiels comportent une indication temporelle. D'autre part, les spécificités temporelles des événements déterminent leur aptitude à servir de repère spatial.

\section{Summary - The spatial denotation of event nouns}

This paper deals with the spatial features of event-denoting nouns [EvNs], which are often overlooked in the linguistic literature on space. EvNs can refer to spatial entities, as they can be used as trajectors in localization sentences (Il y a une cérémonie dans l'église 'There is a ceremony in the church'). Still, EvNs differ in several ways from nouns denoting prototypical spatial entities. They do not combine with complements denoting spatial extension (*une cérémonie de deux hectares 'a four acres' ceremony'), and they are associated with specific nouns and verbs of location (le lieu / *la place de la cérémonie 'the location / the place of the ceremony', Une cérémonie a lieu / *se trouve dans l'église 'A ceremony takes place / is in the church'). It is assumed that the peculiarity of the spatial denotation of EvNs is due to their direct relation to time. The dependence between the spatial and temporal properties of EvNs shows when these nouns are used as landmarks (Pierre se rend à la cérémonie 'Peter goes to the ceremony'). First, spatial eventive landmarks bear a temporal specification. Second, the temporal features of events determine their ability to be used as spatial landmarks. 


\title{
La dénotation spatiale des noms d'événements
}

\author{
Richard Huyghe \\ Université Paris Diderot, EA 3967 CLILLAC-ARP \\ rhuyghe@eila.univ-paris-diderot.fr
}

\section{Introduction}

Dans la plupart des travaux linguistiques consacrés à l'espace, les noms exprimant le localisé (ou cible, relativement à un localisateur donné, le site) décrivent des entités physiques. Sont ainsi considérés comme noms d'entités spatiales des noms d'êtres, d'objets, d'habitations, de territoires, de matières, etc. (cf. L. Talmy 1983, A. Herskovits 1986, C. Vandeloise 1986 et 2001, L. Vieu 1991, S. Svorou 1994, A. Borillo 1998, M. Aurnague 2004 inter alia). De tels noms peuvent figurer en position de cible spatiale dans des phrases thétiques introduites par Il y $a$ :
a. Il y a un homme dans l'entrée
b. Il y a une lampe sur la table
c. Il y a un cèdre dans le jardin
d. Il y a une usine derrière la maison
e. Il y a un champ à côté de chez Pierre
f. Il y a un lac à deux pas du village
g. Il y a de l'eau dans le vase
h. Il y a un espace entre les deux meubles

Les substantifs employés ici pour désigner l'entité localisée décrivent des animés (1a), des objets mobiles (1b) ou fixes (1c-d) dans le référentiel terrestre, des parties du sol terrestre (1e), des étendues d'eau (1f), des matières (1g), des portions d'espace vide (1h).

Cependant, d'autres types de noms peuvent dénoter la cible dans des phrases de localisation spatiale. Tel est le cas dans :
a. Il y a une réunion dans le bureau du directeur
b. Il y a un concert dans le parc
c. Il y a une fête chez les voisins
d. Il y a une cérémonie dans l'église
e. Il y a un examen dans l'amphi
f. Il y a un feu d'artifice sur la plage
g. Il y a une conférence de presse dans le hall de l'hôtel
h. Il y a un match au stade Bollaert

Ces noms diffèrent des précédents en ce qu'ils ne décrivent pas des parties du monde physique. Ils rejettent notamment les compléments de la forme en / de Nmatière (e.g. une lampe en aluminium, un lac de glace vs *une réunion en aluminium, *une cérémonie de glace). On peut les catégoriser comme noms d'événements [Nev], en vertu de leur distribution avec avoir lieu (cf. A. Balibar-Mrabti 1990, G. Gross 1996, D. Godard \& J. Jayez 1996, R. Vivès 2004, R. Huyghe 2009) : 

a. Une réunion a lieu dans le bureau du directeur
b. Un concert a lieu dans le parc
c. Une fête a lieu chez les voisins
d. Une cérémonie a lieu dans l'église

La construction avec avoir lieu sera considérée comme une condition suffisante de l'appartenance d'un nom à la classe des Nev. ${ }^{1}$

Nous nous proposons dans ce travail d'étudier les propriétés descriptives spatiales des Nev, avec en filigrane les questions suivantes : en quoi consiste, d'après la langue, la spatialité des événements ? Les événements, tels qu'ils sont décrits par leurs noms, sont-ils des entités spatiales au même titre que les êtres et les objets ? Les caractéristiques temporelles des événements interagissent-elles avec leurs propriétés spatiales, et si oui, de quelle manière ? Nous voyons dans ce travail un double intérêt. Il s'agit d'une part d'étendre le champ d'étude des expressions spatiales à des formes non prototypiques, et d'autre part d'avancer dans la description et l'analyse des noms d'événements, mieux connus pour leurs propriétés temporelles.

\section{Des cibles spatiales événementielles}

L'idée que les événements sont des entités spatiales est admise par certains philosophes, qui s'interrogent notamment sur la délimitation des sites spatiaux assignés aux événements (cf. D. Davidson 1967 et 1969, A. Quinton 1979, P.M.S. Hacker 1982, R. Casati \& A. Varzi 1999). Mais la question de la spatialité des événements reste peu abordée en linguistique. Pourtant, il existe des indices de la dénotation spatiale des Nev. Outre leur emploi dans les phrases existentielles locatives (2), les noms d'événements peuvent, à l'instar des noms d'entités matérielles, figurer dans la question Où est le $N$ ? (cf. D. Van de Velde 2006 : 186) :

(4) Où est (la réunion / le concert / la fête / le match / le feu d'artifice / la conférence de presse / le mariage / le colloque / l'assemblée générale / le festival)?

Les Nev s'emploient également en complément de localisation du nom lieu, i.e. ils peuvent désigner une cible dans l'expression le lieu $d u N$ (cf. R. Huyghe 2009) :

le lieu (de la réunion / du concert / de la fête / de la cérémonie / de l'examen / de la conférence de presse / de la rencontre / du tournage / de la manifestation / de l'enterrement)

\footnotetext{
${ }^{1}$ Les noms d'événements pris en compte dans cette étude sont ceux qui valident le test avoir lieu, abstraction faite de leur (éventuelle) structure argumentale. Nous ne nous intéresserons pas ici au rôle de la structure argumentale dans la dénotation événementielle, ni au lien sémantique avec le prédicat source en cas de nominalisation - on notera que les Nev considérés ne sont pas tous des déverbaux (e.g. réunion, tournage, manifestation vs cérémonie, festival, colloque). Un travail ultérieur sera consacré à la question de l'autonomie syntaxique des Nev : nous nous interrogerons sur la nécessité pour les différents $\mathrm{Nev}$ d'avoir une structure argumentale, et sur le type d'événement décrit selon le degré d'autonomie du nom.
} 
Lieu s'emploie ici pour dénoter un site de localisation spatiale, associé à une cible événementielle elle-même désignée par le nom en complément.

Les événements sont donc traités linguistiquement comme des entités que l'on peut localiser dans l'espace. En ce sens, les Nev font partie des noms d'entités spatiales. Ce constat appelle toutefois plusieurs remarques.

D'une part, il existe des noms d'événements qui ne valident pas l'ensemble des trois conditions mentionnées ci-dessus. Ainsi la question Où est le $N$ ? ne convientelle pas à certains Nev, pourtant compatibles avec le lieu $d u N$ :

(6) a. le lieu (de l'attentat / de l'avalanche / de l'explosion)

b. *Où est (l'attentat / l'avalanche / l'explosion)?

Les événements en question sont bien des événements spatiaux, mais ils ont certaines particularités qui influent sur l'expression de leur localisation spatiale. Autrement dit, il peut exister des disparités entre les Nev quant à la forme de spatialité décrite. Nous $\mathrm{y}$ reviendrons dans la dernière section.

D'autre part, il faut préciser l'extension de la classe des Nev considérés. En particulier, certains Nev marginaux, appelés noms d' " événements faibles » par D. Godard et J. Jayez (1996) et classés parmi les Nev en vertu de leur compatibilité avec les prépositions temporelles et les constructions nominales de durée (e.g. un $N$ de deux heures), n'entrent dans aucune des trois tournures mentionnées :

a. \#Il y a (une symphonie / une comédie / une chanson) dans le parc

b. \#Où est (la symphonie / la comédie / la chanson)?

c. *le lieu (de la symphonie / de la comédie / de la chanson)

Ces Nev faibles ne sont pas, dans leur emploi événementiel ${ }^{2}$, des noms d'entités spatiales. Cependant, il ne s'agit pas de Nev prototypiques. Incompatibles avec avoir lieu, ils n'accèdent pas à l'individualisation référentielle caractéristique des $\mathrm{Nev}$ du type (2) (D. Godard \& J. Jayez 1996 : 43). Leur absence d'ancrage spatial constitue en ce sens un indice de leur caractère événementiel secondaire.

Ajoutons que certains SN construits autour de Nev forts (i.e. compatibles avec avoir lieu) paraissent moins sensibles que d'autres à l'expression de la localisation spatiale. Ces SN admettent la localisation temporelle, comme l'ensemble des SN événementiels forts, mais ils sont plus rétifs à la localisation spatiale :

a. Quand a eu lieu (la fusion entre les deux entreprises / la cessation de paiement / la suppression de cette aide / la prolongation du congé / le licenciement de Pierre)?

b. ?Où a eu lieu (la fusion entre les deux entreprises / la cessation de paiement / la suppression de cette aide / la prolongation du congé / le licenciement de Pierre)?

\footnotetext{
${ }^{2}$ Comme le notent D. Godard et J. Jayez (1996 : 46), les Nev faibles peuvent se voir associer différents types sélectionnels. Ils admettent notamment l'interprétation concrète d'objet - qu'il s'agisse d'un objet «matériel » (Pierre lui tend la symphonie) ou « informationnel » (Il y a plusieurs idées brillantes dans cette symphonie). Dans cette interprétation d'objet, les Nev faibles peuvent se voir associer une localisation spatiale (La symphonie est posée sur le piano). Cette interprétation concrète est exclue dans le cas qui nous intéresse.
} 
Ce point mériterait de plus amples développements. Il faudrait analyser la structure des SN en question, en tenant compte du degré d'autonomie syntaxique des noms têtes et du caractère physique ou non des événements dénotés, pour le cas échéant envisager l'existence d'une sous-classe de Nev forts non spatiaux.

Nous nous concentrerons pour l'heure sur les noms d'événements dont nous sommes certain qu'ils dénotent des entités spatiales, en l'occurrence sur les Nev forts qui peuvent se construire avec lieu dans le lieu $d u N$. Nous verrons que ces Nev décrivent des entités spatiales d'un autre type que celles dénotées dans (1). L'hypothèse défendue sera que leur rapport à l'espace est différent car leur rapport au temps l'est aussi.

\section{Absence d'extension spatiale}

La dénotation spatiale des Nev se distingue de celle de noms comme homme, lampe, usine, champ, lac, etc. par plusieurs aspects. Une première différence est que les Nev se combinent difficilement avec les expressions décrivant l'étendue spatiale. En effet, contrairement aux noms d'entités matérielles, les Nev rejettent les compléments de mesure spatiale en de:
a. un homme de deux mètres
b. une lampe de trente centimètres de haut
c. un champ de soixante hectares
a. *une réunion de trente mètres carrés
b. *un concert de cinquante mètres de large
c. *une cérémonie de mille mètres carrés ${ }^{3}$

Les Nev ne sont pas non plus sujet de mesurer / faire + mesure spatiale :

(11) a. Ce cèdre fait vingt mètres de haut

b. L'usine fait quinze mille mètres carrés

c. Ce lac fait trois mille kilomètres carrés
a. *La conférence de presse fait deux cents mètres carrés
b. *Le feu d'artifice fait cent mètres de haut
c. *Le mariage fait deux hectares
a. L'ampoule mesure cinq centimètres de diamètre
b. Sophie mesure un mètre soixante-dix
c. Un terrain de foot mesure cent mètres de longueur

\footnotetext{
${ }^{3}$ Font exception à ce principe des noms comme course, randonnée, marche, etc., puisqu'il est possible de dire une (course / marche / randonnée) de vingt kilomètres. Cependant, le complément de mesure peut être considéré ici comme l'argument interne d'une nominalisation d'un verbe de déplacement (courir vingt kilomètres $>$ une course de vingt kilomètres). Autrement dit, la mesure s'applique à un processus et non à un objet, ce qui distingue une course de vingt kilomètres de une piste de vingt kilomètres par exemple. Seule la première expression est compatible avec un verbe support dynamique (effectuer (une course / *une piste) de vingt kilomètres) et se paraphrase dans cet emploi par effectuer vingt kilomètres en courant.
} 

a. *L'examen mesure dix mètres
b. *La fête mesure cent mètres de diamètre
c. *Un match de foot mesure cent mètres de longueur

En outre, les Nev ne peuvent pas compléter des noms de dimensions spatiales, tels que longueur, largeur, hauteur, superficie, dimension, etc. :

(15) les dimensions de la lampe, la hauteur de l'usine, la superficie du champ, la largeur du terrain, la circonférence du lac

*les dimensions du concert, *la hauteur du feu d'artifice, *la superficie de la réunion, *la largeur du match, *la circonférence de la fête

Les événements, tels qu'ils sont décrits par leurs noms, ne se voient donc pas directement attribuer d'extension spatiale. Ils se présentent comme des entités adimensionnelles. Il serait toutefois abusif d'en conclure que les événements ne sont pas des entités spatiales, puisqu'ils peuvent faire l'objet d'une localisation dans l'espace. Il est nécessaire de distinguer dans l'ordre de la spatialité les propriétés d'étendue et les propriétés d'ancrage — c'est leur assimilation implicite qui conduit certains auteurs (e.g. Z. Vendler 1967) à considérer les événements comme des entités essentiellement temporelles, et non directement spatiales. Au niveau des formes linguistiques, l'absence d'extension n'implique pas l'absence de localisation. ${ }^{4}$

Propriétés d'extension et de localisation spatiales, si elles doivent être distinguées, ne sont pas pour autant cloisonnées. Comme nous allons le voir, le fait pour une cible donnée d'avoir ou non une étendue peut déterminer son mode de localisation.

\section{Différentes formes de localisation spatiale}

L'adimensionnalité des événements a pour conséquence qu'ils ne mobilisent pas l'étendue spatiale. De fait, les Nev, contrairement aux noms d'êtres et d'objets, sont difficilement compatibles avec prendre de la place $(+$ loc) :

a. Cette lampe prend de la place (sur le bureau)

b. Le cèdre prend de la place (dans le jardin)

c. Pierre prend de la place (dans le fauteuil)

a. ?? Ce concert prend de la place (dans le parc)

b. ?? La fête prend de la place (à l'étage du dessus)

c. ??Le feu d'artifice prend de la place (sur la plage)

Place, par son caractère massif, met en profil l'étendue spatiale, en tant qu'elle peut être saturée par un objet (cf. J.-J. Franckel 1993, C. Vandeloise 2001, R. Huyghe 2009). L'interprétation de prendre de la place comme " prendre beaucoup de place » est d'ailleurs souvent permise. Place dans (17) indique un schéma d'occupation de

\footnotetext{
${ }^{4}$ Il en va de même dans le temps que dans l'espace, puisque certains événements, conçus comme ponctuels, sont tout à fait datables (e.g. *une naissance de deux heures vs la date de la naissance, cf. P. Haas et al. 2008).
} 
l'espace, i.e. de mobilisation exclusive de l'étendue par une cible. Ce schéma ne s'applique pas aux Nev, car ils décrivent des entités sans extension. Comme le montre P.M.S. Hacker (1982), tenter de définir précisément la portion d'espace occupée par un événement, par exemple la place que prend le mariage dans l'église ou la place que prend la chute de Pierre dans l'escalier, n'est pas pertinent du point de vue de l'usage linguistique.

On a donc affaire avec les objets et les événements à des façons d'être dans l'espace différentes. La forme de localisation varie, selon que la mobilisation de l'étendue est ou non impliquée. Cette différence s'exprime de deux façons, par des noms et des verbes de localisation distincts.

Les noms d'êtres ou d'objets peuvent apparaître en complément de localisation du nom place mais pas de lieu. Inversement, les noms d'événements s'emploient avec lieu mais pas avec place ${ }^{5}$ :

(19) (la place / *le lieu) de Marion, (la place / *le lieu) du chien, (la place / *le lieu) de la lampe, (la place / *le lieu) de l'ordinateur, (la place / *le lieu) de l'armoire, (la place / *le lieu) des disques

(20) (le lieu / *la place) de la cérémonie, (le lieu / *la place) de la manifestation, (le lieu / *la place) du tournage, (le lieu / *la place) du rendez-vous, (le lieu / *la place) du meeting, (le lieu / *la place) du rassemblement

Place et lieu sont associés à des formes de localisation différentes (cf. R. Huyghe 2009). Place, comme on l'a vu dans prendre de la place, indique une mobilisation de l'étendue. La forme de localisation est substantielle, en ce sens que place décrit un espace voué à être occupé par une entité autonome — d'où la sélection de noms d'êtres ou d'objets. Lieu au contraire indique dans ses emplois distinctifs une localisation processive, i.e. une localisation qui s'accompagne de la réalisation de quelque chose, comme en témoignent également les expressions de la forme lieu de Nactivité (un lieu de travail, un lieu de méditation, un lieu d'entraînement, etc.). Avec lieu, l'espace n'est pas envisagé comme un réceptacle, mais comme un cadre d'action, d'où la sélection de noms de cibles événementielles.

Les verbes employés pour introduire la localisation spatiale diffèrent également : les noms d'êtres et de choses se construisent avec se trouver, alors que les noms d'événements sont sujet de avoir lieu. L'inverse n'est pas permis (cf. D. Godard \& J. Jayez 1996, R. Huyghe 2009) :
a. Pierre (se trouve / *a lieu) à Helsinki
b. Ce livre (se trouve / *a lieu) sur l'étagère
c. Un cèdre (se trouve / *a lieu) à l'entrée du parc
d. L'université (se trouve / *a lieu) dans le centre ville

\footnotetext{
${ }^{5}$ Place n'est considéré ici que dans son sens général de localisateur spatial. Le sens de «place publique » est exclu, de même que les emplois dans des univers de référence non spatiaux (e.g. la place du mariage dans la société française).
} 
(22) a. Ce spectacle (a lieu / *se trouve) à la salle des fêtes de Douai

b. Un meeting (a lieu / *se trouve) place de la République

c. L'avant-première (a lieu / *se trouve) dans une salle d'art et d'essai

d. Un rassemblement (a lieu / *se trouve) devant le ministère

La différence sémantique entre les deux verbes est du même type que celle observée pour lieu / place : avoir lieu sélectionne des cibles dynamiques, se trouver des cibles substantielles. En effet, avoir lieu se distingue de se trouver par le fait qu'il exprime l'actualisation de quelque chose. L'emploi sans complément de localisation est possible :

(23) a. Ce spectacle a (effectivement) eu lieu

b. La rencontre entre les deux hommes a (effectivement) eu lieu

c. Cette conversation a (effectivement) eu lieu
a. *Pierre se trouve (effectivement)
b. ${ }^{*}$ L'armoire se trouve (effectivement)
c. *' 'usine se trouve (effectivement)

Alors que se trouver est un verbe de pure localisation, avoir lieu dans (22) lie la localisation de la cible à sa réalisation, présupposant ainsi la dynamicité du sujet. Une phrase de la forme $X$ a eu lieu à tel endroit comporte en fait deux informations : (i) $\mathrm{X}$ s'est réalisé et (ii) $\mathrm{X}$ est localisé à tel endroit. Dans le cas d'avoir lieu, l'ancrage spatial se combine donc avec une actualisation processive.

\section{Une dénotation spatiale et temporelle}

Le traitement linguistique de la localisation diffère selon que les cibles sont désignées par des noms d'événements ou d'entités matérielles. La forme de localisation particulière associée aux Nev met en jeu la nature dynamique des entités dénotées, et donc leur rapport au temps. La dynamicité implique en effet une relation directe au temps, qui échappe aux entités matérielles.

De fait, une autre différence entre avoir lieu et se trouver est que avoir lieu introduit indifféremment un complément de lieu ou de temps, alors que se trouver convoque nécessairement un complément de lieu. En effet, se trouver ne se satisfait pas du seul constituant de localisation temporelle :

a. La cérémonie a lieu à Paris

b. La cérémonie a lieu cet après-midi

c. La cérémonie a lieu à Paris cet après-midi

a. Pierre se trouve à Paris

b. *Pierre se trouve cet après-midi

c. Pierre se trouve à Paris cet après-midi

Le même constat vaut en cas de substitution du verbe être à avoir lieu / se trouver : 
a. La cérémonie est à Paris

b. La cérémonie est cet après-midi

c. La cérémonie est à Paris cet après-midi

a. Pierre est à Paris

b. *Pierre est cet après-midi

c. Pierre est à Paris cet après-midi

Les événements peuvent se voir associer un ancrage temporel direct. Dans le cas des entités matérielles par contre, l'ancrage temporel est subordonné à la localisation spatiale. Dans (26c) et (28c), la localisation temporelle s'applique à une situation spatiale, celle dénotée par Pierre est / se trouve à Paris, et non directement au sujet Pierre. Autrement dit, le constituant temporel dans ces phrases est un adjoint tandis que le constituant spatial ressortit au prédicat de phrase. Il n'y a rien de tel dans $(25 \mathrm{c})$ et $(27 \mathrm{c})$, où compléments de lieu et de temps sont sur un pied d'égalité syntaxique. Cette différence de niveau de localisation témoigne du fait que, contrairement aux noms d'êtres et d'objets, les Nev décrivent des entités temporelles, i.e. des entités directement inscrites dans le temps.

La dénotation temporelle des Nev se trouve confirmée par leur compatibilité avec les prépositions ou locutions prépositionnelles temporelles (cf. Z. Vendler 1967, A.M. Berthonneau 1989, D. Godard \& J. Jayez 1996) :

(29) a. pendant la manifestation, lors de la cérémonie, durant la réunion, au cours de la conversation, au moment du tournage

b. *pendant Pierre, *lors du chien, *durant l'usine, *au cours $d u$ cèdre, *au moment de la table ${ }^{6}$

Les Nev décrivent donc des entités à la fois spatiales et temporelles, contrairement aux noms d'entités matérielles. Notre hypothèse est que cette double nature explique la particularité de la dénotation spatiale des Nev, autrement dit, que la différence de référence spatiale entre noms d'événements et noms d'entités matérielles est corrélée à leur différence de référence temporelle. Dans le cas des événements, le fait d'avoir un rapport au temps conditionne une forme de spatialité non prototypique.

\section{Les noms d'événements en position de site}

Le fait que les Nev dénotent des entités spatiales et temporelles et l'imbrication de ces deux dénotations apparaissent dans leur emploi en position de site.

\footnotetext{
${ }^{6}$ Il existe des cas où la préposition temporelle peut se distribuer avec des noms d'êtres ou d'objets, par exemple dans Ne laisse pas les gens entrer pendant les tigres (A.-M. Berthonneau 1989 : 681) et Elle est partie avant le fromage (A. Le Draoulec \& C. Fabre 2006 : 55). Néanmoins, comme le montrent A.-M. Berthonneau, A. Le Draoulec et C. Fabre, l'emploi de la préposition temporelle impose dans ce genre de cas que l'on associe un événement au nom d'objet sélectionné. La localisation temporelle signifiée par le SP s'établit relativement à cet événement.
} 


\subsection{Une localisation spatio-temporelle}

A ce stade, nous n'avons considéré les événements qu'en tant que cibles de localisation. Mais ils peuvent également servir de repères : les Nev peuvent se trouver en position de site, notamment dans des SP introduits par $\grave{a}$. Ils indiquent alors une localisation spatio-temporelle :

(30) a. Pierre et Vincent se sont vus au concert de Kurt Wagner

b. Les deux réalisateurs se sont rencontrés à la cérémonie des Oscars

c. Le ministre a prononcé ce discours à la réunion des députés d'Outre-Mer

On peut par abstraction isoler la composante spatiale ou temporelle de cette double localisation. En effet, les phrases sous (30) répondent aussi bien à la question Où... ? qu'à la question Quand... ? :

(31) a. Où Pierre et Vincent se sont-ils vus? - Au concert de Kurt Wagner

b. Quand Pierre et Vincent se sont-ils vus ? - Au concert de Kurt Wagner

(32) a. Où les deux réalisateurs se sont-ils rencontrés ? - A la cérémonie des Oscars

b. Quand les deux réalisateurs se sont-ils rencontrés ? - A la cérémonie des Oscars

(33) a. Où le ministre a-t-il prononcé ce discours ? - A la réunion des députés d'Outre-Mer

b. Quand le ministre a-t-il prononcé ce discours ? - A la réunion des députés d'Outre-Mer

Dans (30), les deux informations, spatiale et temporelle, sont intimement imbriquées. Ce type de localisation s'exprime idéalement avec la préposition à. Celle-ci en effet s'applique à la fois dans les domaines spatial (à Paris) et temporel (à quatre heures). Elle indique de plus une coïncidence sans spécifier de configuration, ce qui convient bien à l'adimensionnalité des événements — plus rétifs aux contraintes imposées par l'emploi des prépositions spatiales dans, sur, sous, au-dessus de, etc. ${ }^{7}$

La référence spatio-temporelle des Nev apparaît également dans leurs emplois comme stricts constituants de lieu, par exemple avec des verbes de déplacement :
a. Pierre va au match France-Ecosse
b. Pierre se rend à la conférence de presse du maire
c. Pierre est allé au mariage de son collègue

Le Nev figure ici syntaxiquement en position de lieu, puisqu'on peut dire (35a) et non $(35 b)$ :

\footnotetext{
${ }^{7}$ L'emploi des Nev avec dans et sur n'est toutefois pas exclue (e.g. Ils se sont rencontrés dans un concert / sur un tournage). Il serait intéressant d'analyser la variation du sens de la préposition selon le type de $\mathrm{N}$ sélectionné (objet $v s$ événement) - les descriptions spatiales en termes de site « contenant» ou « porteur » (C. Vandeloise 1986) n'étant pas pertinentes lorsque l'argument de la préposition est un Nev.
} 
Il n'en reste pas moins que, même dans des phrases comme (34), le Nev donne une indication temporelle. En effet la différence entre (34) et :

\section{Pierre va au Jardin des Plantes}

est que le site dans (34) détermine le moment du déplacement, qui correspond à la phase de réalisation des événements désignés. Ainsi (34a) signifie-t-il que Pierre va à l'endroit où a lieu le match, au moment où a lieu le match. La présence d'une indication temporelle distingue (34) de (37) :

(37) a. Pierre va sur le lieu du match France-Ecosse

b. Pierre se rend sur le lieu de la conférence de presse du maire

c. Pierre est allé sur le lieu du mariage de son collègue

Le Nev n'est pas paraphrasable par le lieu du Nev, précisément parce que le Nev implique une spécification temporelle. Contrairement au localisateur dans (36), le site dans (34) ne se réduit pas à un lieu. En témoigne également l'impossibilité de reprise par cet endroit :

(38) Pierre va au Jardin des Plantes. Il trouve cet endroit incroyable

a. Pierre va au match France-Ecosse. *Il trouve cet endroit incroyable

b. Pierre se rend à la conférence de presse du ministre des affaires étrangères. *Cet endroit est bondé

c. Pierre est allé au mariage de son collègue. ${ }^{*}$ Cet endroit était très accueillant

L'anaphore dans (39) est exclue car endroit établit un repère spatial hors temps; or le $\mathrm{Nev}$ conserve ses propriétés descriptives temporelles. La finitude et l'ancrage temporels associés aux Nev ne leur permet pas de désigner de purs sites spatiaux, contrairement à ce qui est le cas pour les noms d'entités matérielles.

Le fait que les Nev soient dotés de traits temporels les empêche donc de désigner des repères spatiaux au même titre que les noms d'objets. Leur rapport au temps altère leur dénotation spatiale.

\subsection{Evénements sans fonction de repère spatial}

La capacité des Nev à indiquer une localisation à la fois temporelle et spatiale doit être nuancée. En effet, parmi les noms d'événements spatiaux (i.e. compatibles avec lieu $d u N$ ), certains n'apparaissent en position de site que dans des emplois temporels. La fonction de repérage spatial est pour eux exclue. En position d'adjoint, ces noms s'emploient difficilement avec $a ̀$, privilégiant les prépositions strictement temporelles : 
(40) a. Les deux banquiers se sont rencontrés (??à / au cours d') une transaction délicate

b. Vincent et Sophie se sont vus (??à / lors de) la condamnation de leur père

c. Les généraux se sont parlé (??à / pendant) l'exécution des prisonniers politiques

De même, certains Nev ne s'emploient pas avec les verbes de déplacement :

(41) a. ??Marianne va à l'enquête sur la mort de sa meilleure amie

b. ??Pierre se rend au recrutement du nouveau directeur adjoint

c. ??Il est allé au dépannage de l'ascenseur

Par ailleurs, l'emploi comme localisateur spatial peut dépendre de la construction dans laquelle apparaît le Nev. La détermination, les spécificités des arguments, la possibilité d'associer un lieu fixe à l'événement semblent jouer un rôle dans la dénotation de repères spatiaux :

(42) a. Pierre va (à une vente d'objets anciens / ??à la vente de sa maison)

b. Les journalistes se rendent (au discours d'investiture du président / ??à un discours important du ministre des finances)

Une étude approfondie serait nécessaire pour rendre compte des contraintes complexes qui déterminent le type de localisation décrit par les Nev en position de site. On peut cependant noter d'emblée certaines régularités.

Parmi les Nev qui récusent l'emploi comme localisateur spatial figure un sousensemble homogène de noms dénotant des événements non contrôlés. Il s'agit de Nev tels que :

(43) attentat, avalanche, crash, séisme, assassinat, drame, crime, explosion, catastrophe, coup d'état, collision, noyade, secousse, choc, déraillement, miracle, suicide, meurtre, débordement, altercation, massacre, déluge, décès, infraction, agression, révolte, attaque, délit, mutinerie, dispute, effraction

Ces noms, bien que renvoyant à des entités ancrées dans l'espace - ils sont compatibles avec lieu —, ne peuvent dénoter des repères spatiaux ni en position d'adjoint ni en position d'argument :

*Les deux hommes se sont rencontrés (à l'attentat / à l'avalanche / au crash du Concorde / etc.)

(45) a. *Pierre est allé (au séisme / à l'assassinat du juge / au drame / etc.)

b. *Pierre s'est rendu (au crime / à l'explosion / à la catastrophe / etc.)

Les Nev sous (43) ont pour caractéristique de décrire des événements qui surviennent de façon imprévue et que l'on constate généralement après coup. Contrairement aux Nev considérés en 5.1., ils s'emploient avec se produire : 
(46) a. Un drame s'est produit à la gare de Namur

b. Une explosion s'est produite dans une résidence universitaire de Montpellier

c. Un coup d'état s'est produit au Niger

d. Une collision s'est produite entre deux pétroliers au large de Singapour

e. Une noyade s'est produite sur la plage de Valras

f. Une secousse s'est produite dans l'est de la capitale

Les exemples (46) contrastent en effet avec les suivants :

(47) a. *Une réunion s'est produite dans le bureau du directeur

b. *Une fête s'est produite chez les voisins

c. *Un concert s'est produit dans le parc

d. *Un colloque de linguistique s'est produit à Gabès

e. *Un mariage s'est produit dans la salle des fêtes

f. *Un feu d'artifice s'est produit sur la plage

Se produire est plus sélectif qu'avoir lieu, qui accepte l'ensemble des Nev sous (46)(47). Se produire exprime la réalisation d'un événement imprévu, d'où une certaine prédilection pour les noms d'événements naturels (cf. D. Godard \& J. Jayez 1996 : 43). Les événements impliquant des acteurs humains sont également admis, pour peu qu'ils se présentent comme accidentels, c'est-à-dire comme non programmés par l'ensemble de ceux qui y prennent part (cf. G. Gross 1996 : 60). Le point commun entre tous les Nev compatibles avec se produire est de décrire des événements qui, d'une façon ou d'une autre, échappent à notre contrôle.

Non prévus et non contrôlés, ces événements sont souvent constatés après qu'ils se sont réalisés. De fait, la plupart des Nev sous (43) privilégient les constructions aux temps accomplis, notamment dans leur emploi avec Il y a (48)-(50) et avoir lieu (51)-(53) :

(48) a. Il y a un attentat au Caire (-)

b. Il y a eu un attentat au Caire $(+)$

(49) a. Il y a une avalanche au Grand Ballon d'Alsace (-)

b. Il y a eu une avalanche au Grand Ballon d'Alsace (+)

(50) a. Il y a un choc terrible entre les deux véhicules (-)

b. Il y a eu un choc terrible entre les deux véhicules $(+)$

(51) a. Un déraillement a lieu sur la ligne Paris-Brest (-)

b. Un déraillement a eu lieu sur la ligne Paris-Brest $(+)$

(52) a. Un miracle a lieu à Faverney (-)

b. Un miracle a eu lieu à Faverney (+)

(53) a. Un suicide a lieu à l'usine PSA de Mulhouse (-)

b. Un suicide a eu lieu à l'usine PSA de Mulhouse (+) 
De même, ces Nev s'emploient difficilement dans Où est le $N$ ? en référence à une localisation spatiale (cf. exemple (6b)). Ainsi oppose-t-on (4) et (54) :

Où est (la réunion / le concert / la fête / le match / le feu d'artifice / la conférence de presse / le mariage / le colloque / l'assemblée générale / le festival)?

*Où est (l'avalanche / la collision / l'explosion / le déraillement / le meurtre / le débordement / l'altercation / le massacre / le déluge / le décès) $?^{8}$

Les événements de (48)-(54) ne s'installent pas dans le temps présent. Beaucoup d'entre eux se voient difficilement associer une durée (e.g. ?? un attentat de deux heures, ?? un meurtre de dix minutes, cf. R. Huyghe \& R. Marín 2008). Aussi, en pratique, ne constituent-ils pas des repères spatiaux performants. Le fait qu'ils surviennent de façon impromptue et fugitive entrave leur stabilité spatiale et les empêche de constituer des sites spatiaux efficaces, pouvant faire l'objet de projections de localisation.

Une règle se dessine ici : moins un événement est contrôlé, stable et programmé dans le temps, moins il a de chances de constituer un bon repère spatial. On constate de nouveau que la spatialité des événements dépend étroitement de leur temporalité. Les spécificités aspectuo-temporelles des événements déterminent leur capacité à servir de site spatial.

\section{Conclusion}

Il y a des Nev qui décrivent des entités spatiales, i.e. des entités susceptibles de constituer des cibles de localisation spatiale. Ces Nev ne sont toutefois pas des noms d'entités spatiales au même titre que les noms d'êtres et d'objets. Ils ne se combinent pas avec les compléments d'étendue spatiale et se construisent avec des noms et des verbes de localisation qui leur sont propres. On leur associe un mode de localisation particulier, qui n'envisage pas l'espace de localisation comme un espace à occuper, mais comme un cadre d'action. Ces particularités linguistiques confortent l'idée selon laquelle les événements sont « des entités spatiales d'un autre type que les objets matériels » (R. Casati \& A. Varzi 1999 : 6-7).

La spécificité descriptive spatiale des $\mathrm{Nev}$ tient essentiellement à une relation directe avec le temps. Les Nev décrivent primitivement des entités spatiotemporelles, et non uniquement spatiales, si bien que la dénotation spatiale des Nev est intimement liée à leur dénotation temporelle. C'est cette dépendance qui

\footnotetext{
${ }^{8}$ Certains noms d'événements non contrôlés, comme éboulement, hémorragie, inondation, peuvent se construire dans cette dernière tournure (Où est (l'éboulement / l'hémorragie / l'inondation) ?). On peut toutefois se demander si c'est l'événement à proprement parler qui fait ici l'objet d'une localisation, ou son résultat - en l'occurrence, les dégâts causés par l'action. L'alternance avec avoir lieu, qui sélectionne l'interprétation purement événementielle, atteste d'un emploi au présent peu naturel : ??Où a lieu (l'éboulement / l'hémorragie / l'inondation) ? vs Où a eu lieu (l'éboulement / l'hémorragie / l'inondation) ? A titre de comparaison, la substitution de a lieu à est se fait sans difficulté pour les noms d'événements contrôlés (Où (est / a lieu) (la réunion / le concert / la fête / etc.) ?).
} 
détermine une forme de spatialité particulière, non prototypique — en ce sens que les événements ne relèvent pas de l'espace « pur », considéré en dehors de toute variable temporelle.

En outre, il y a à l'intérieur de la classe des Nev des degrés de spatialité, qui apparaissent dans la capacité de ces noms à s'employer comme complément de lieu, et qui sont eux aussi, en partie au moins, conditionnés par les caractéristiques temporelles des Nev. Plus un événement est stable dans le temps (i.e. contrôlé, projeté et duratif), plus son empreinte spatiale paraît forte. Ainsi, des rapports au temps différents déterminent des rapports à l'espace différents, ce qui témoigne à nouveau de l'influence de la temporalité des événements sur leur spatialité.

Corollairement, le critère spatial peut être un paramètre de discrimination de différents types événementiels. Il est en effet possible, par la mise en évidence de différents niveaux de référence spatiale, d'étayer la distinction entre plusieurs sortes d'événements.

\section{Références}

Aurnague, Michel. 2004. Les structures de l'espace linguistique. Regards croisés sur quelques constructions spatiales du basque et du français. Louvain / Paris : Editions Peeters.

Balibar-Mrabti, Antoinette. 1990. Analyse d'adverbes en dans. Langue Française 86, Paris : Larousse.

Berthonneau, Anne-Marie. 1989. Composantes linguistiques de la référence temporelle. Les compléments de temps, du lexique à l'énoncé. Thèse de doctorat d'état, Université de Paris VII.

Borillo, Andrée. 1998. L'espace et son expression en français. Paris : Ophrys.

Casati, Roberto ; Achille Varzi. 1999. Parts and Places. The Structures of Spatial Representation. Cambridge Mass. : The MIT Press.

Davidson, Donald. 1967 [1993]. La forme logique des phrases d'action. In Actions et événements, Paris : PUF.

Davidson, Donald. 1969 [1993]. L’individuation des événements. In Actions et événements, Paris : PUF.

Franckel, Jean-Jacques. 1993. Il y a lieu de prendre place dans un endroit facilement localisable. In Opérations énonciatives et interprétation de l'énoncé. Mélanges offerts à Janine Bouscaren, L. Danon-Boileau, J.-L. Duchet (éds), Paris : Ophrys.

Godard, Danièle ; Jacques Jayez. 1996. Types nominaux et anaphores : le cas des objets et des événements. In Anaphores temporelles et (in-)coherence, W. De Mulder, L. Tasmowski-De Ryck, C. Vetters (éds), Cahiers Chronos 1, Amsterdam : Rodopi.

Gross, Gaston. 1996. Prédicats nominaux et compatibilité aspectuelle. Langages 121, Paris : Larousse.

Haas, Pauline ; Richard Huyghe ; Rafael Marín. 2008. Du verbe au nom : calques et décalages aspectuels. In Congrès Mondial de Linguistique Française - CMLF'08, J. Durand, B. Habert, B. Laks (éds), Paris : Institut de Linguistique Française.

Hacker, Peter Michael Stephan. 1982. Events and objects in space and time. Mind 91, Oxford : Oxford University Press.

Herskovits, Annette. 1986. Language and Spatial Cognition. An Interdisciplinary Study of the Prepositions in English. Cambridge : Cambridge University Press.

Huyghe, Richard. 2009. Les noms généraux d'espace en français. Enquête linguistique sur la notion de lieu. Bruxelles : De Boeck Duculot.

Huyghe, Richard ; Rafael Marín. 2008. L’héritage aspectuel des noms déverbaux en français et en espagnol. Faits de Langue 30, Paris : Ophrys.

Le Draoulec, Anne ; Cécile Fabre. 2006. La dimension événementielle du syntagme nominal dans la structure avant + SN. Cahiers de Lexicologie 89-2, Paris : Honoré Champion.

Quinton, Anthony. 1979. Objects and events. Mind 88, Oxford : Oxford University Press. 
Svorou, Soteria. 1994. The Grammar of Space. Amsterdam / Philadelphia : John Benjamins Publishing Company.

Talmy, Leonard. 1983. How language structures space. In Spatial Orientation. Theory, Research and Application, H.L. Pick, L.P. Acredolo (éds), New York : Plenum Press.

Vandeloise, Claude. 1986. L'espace en français. Paris : Editions du Seuil.

Vandeloise, Claude. 2001. Aristote et le lexique de l'espace. Rencontres entre la physique grecque et la linguistique cognitive. Stanford : CSLI Publications.

Van de Velde, Danièle. 2006. Grammaire des événements. Villeneuve d'Ascq : Presses Universitaires du Septentrion.

Vendler, Zeno. 1967. Facts and Events. In Linguistics in Philosophy, Ithaca N.Y. : Cornell University Press.

Vieu, Laure. 1991. Sémantique des relations spatiales et inférences spatio-temporelles. Une contribution à l'étude des structures formelles de l'espace en langage naturel. Thèse de doctorat, Université Paul Sabatier, Toulouse.

Vivès, Robert. 2004. Une grille d'analyse pour les prédicats nominaux. In Lexique, Syntaxe et Lexique-Grammaire / Syntax, Lexis \& Lexicon-Grammar. Papers in honour of Maurice Gross, C. Leclère, E. Laporte, M. Piot, M. Silberztein (éds), Lingvisticae Investigationes Supplementa 24, Amsterdam / Philadelphia : John Benjamins Publishing Company. 